negative smear or culture sample sent in the last month of treatment) as a successful outcome. This could be emphasised in future guidelines, with the proviso that if there is no proof of bacteriological cure, greater efforts should be made to improve initial microbiological TB diagnosis. This would enable individualised patient-specific drug susceptibility results to inform regimen choice and ensure that treatment completion is a robust indicator of success. In light of the new information from this study, we would suggest similar studies are performed in different settings both in the UK and elsewhere. The information obtained may allow for better selection of patients to be tested at treatment completion.

0 @ERSpublications

In UK hospitals obtaining evidence of TB cure is impractical and unnecessary in the majority of treated individuals http://ow.ly/mxhQM

Abbey Ursula Leahy ${ }^{1}$, Marc Lipman ${ }^{2}$, Martin Hetzel ${ }^{1}$, Onn Min Kon ${ }^{3}$, Susan Hopkins ${ }^{2}$ and Ibrahim Abubakar ${ }^{4}$ ${ }^{1}$ University Hospitals Bristol NHS Foundation Trust, Bristol, ${ }^{2}$ Royal Free London NHS Foundation Trust and University College London, London, ${ }^{3}$ Imperial College Healthcare NHS Trust and National Heart and Lung Institute, London, and

${ }^{4}$ Tuberculosis Section, Health Protection Agency Colindale and University College London, London, UK.

Correspondence: I. Abubakar, Tuberculosis Section, Health Protection Agency Colindale and University College London, London, UK. E-mail: i.abubakar@ucl.ac.uk

Received: Nov 252012 | Accepted after revision: March 022012

Conflict of interest: None declared.

\title{
References
}

1 European Centre for Disease Prevention and Control. Progressing towards TB elimination. A follow-up to the Framework Action Plan to Fight Tuberculosis in the European Union. Stockholm, ECDC, 2010.

2 Department of Health. Stopping Tuberculosis in England: An action plan from the Chief Medical Officer. http://webarchive.nationalarchives.gov.uk/20130107105354/http://www.dh.gov.uk/prod_consum_dh/groups/dh_ digitalassets/@dh/@en/documents/digitalasset/dh_4100860.pdf Date last accessed: July 3, 2013. Date last updated: October 7, 2004

3 Health Protection Agency. Tuberculosis in the UK: 2011 report. London, HPA, 2011.

4 Veen J, Migliori GB, Raviglione M, et al. Harmonisation of TB control in the WHO European region: the history of the Wolfheze Workshops. Eur Respir J 2011; 37: 950-959.

5 Migliori GB, Zellweger JP, Abubakar I, et al. European union standards for tuberculosis care. Eur Respir J 2012; 39: 807-819.

6 Veen J, Raviglione M, Rieder HL, et al. Standardized tuberculosis treatment outcome monitoring in Europe. Recommendations of a Working Group of the World Health Organization (WHO) and the European Region of the International Union Against Tuberculosis and Lung Disease (IUATLD) for uniform reporting by cohort analysis of treatment outcome in tuberculosis patients. Eur Respir J 1998; 12: 505-510.

7 Falzon D, Jaramillo E, Schünemann HJ, et al. WHO guidelines for the programmatic management of drug-resistant tuberculosis: 2011 update. Eur Respir J 2011; 38: 516-528.

8 Vats A, Vincent CA, Nagpal K, et al. Practical challenges of introducing WHO surgical checklist: UK pilot experience. BMJ 2010; 340: b5433.

9 Crofts JP, Andrews NJ, Barker RD, et al. Risk factors for recurrent tuberculosis in England and Wales, 1998-2005. Thorax 2010; 65: 310-314.

\section{Adherence to positive airway pressure in non-sleepy patients with obstructive sleep apnoea}

\section{To the Editor:}

Complaints of excessive daytime sleepiness (EDS) are absent in many individuals with obstructive sleep apnoea (OSA). The influence of EDS prior to treatment on continuous positive airway pressure (CPAP) adherence has not been clearly determined $[1,2]$. The aim of this prospective cohort study was to evaluate the adherence and perceived benefit during long-term CPAP therapy in a "real life" population of nonsleepy OSA patients. 
Since May 15, 2007 consecutive patients investigated in seven sites in the west of France for symptoms of OSA (snoring, breathing pauses during sleep, morning headaches and EDS) have been recruited in the Institut de Recherche en Santé Respiratoire (IRSR) sleep cohort [2]. Patients filled in questionnaires including the Epworth sleepiness scale (ESS), the Pichot depression scale, the Medical Outcomes Study 36item short-form (SF-36) for health-related quality of life (HRQoL), and questionnaires evaluating socioeconomic status (SES) [2]. As previously described [3], patients also completed surveys including anthropomorphic data and comorbidities. The study was approved by the University of Angers ethics committee and all patients gave their written informed consent. Between May 15, 2007 and May 15, 2012, we enrolled 4660 patients with an apnoea/hypopnoea index (AHI) $\geqslant 5$ on sleep recording (overnight polysomnography or respiratory recording, performed as previously described [3]) of whom 1477 (31.7\%) were non-sleepy (ESS $<11$ and no complaint of EDS) and $3183(68.3 \%)$ were sleepy (ESS $\geqslant 11$ or a report of frequently feeling unrested or sleepy). When compared with sleepy patients, non-sleepy patients were less likely to use anxiolytics, to be depressed or diabetic, had lower body mass index (BMI) and AHI. The percentage of patients with an education level $\leqslant$ high school was higher in non-sleepy patients. On multivariable logistic regression analysis, including age, sex, BMI, AHI, depression, diabetes and level of education, only depression, AHI and level of education were independently associated with EDS. Adjusted odds ratios (95\% confidence intervals) for being sleepy were 2.29 (1.97-2.68) for depression, 1.38 (1.21$1.58)$ for $\mathrm{AHI} \geqslant 30$ versus $<30$ and $0.81(0.71-0.94)$ for a level of education $\leqslant$ high school.

Among 2648 consecutive patients who were prescribed CPAP (fixed CPAP n=980, or autoCPAP $n=1668$; see [2] for details on CPAP initiation and follow-up) according to the French guidelines (AHI $\geqslant 30$ regardless of symptoms or AHI $\geqslant 5$ and $<30$ in combination with EDS that cannot be better explained by other factors or severe cardiovascular morbidity), 125 refused treatment, 135 were lost to follow-up and 2388 had available follow-up data, including 589 (24.7\%) non-sleepy and 1799 (75.3\%) sleepy patients of similar OSA severity (mean \pm SD AHI $40.8 \pm 19.0$ and $41.0 \pm 22.5$, respectively). The mean follow-up after initiating CPAP was $623 \pm 456$ days. Data from questionnaires at diagnosis and during CPAP therapy at the most recent clinic visit are presented in table 1. In both sleepy and non-sleepy patients, CPAP therapy was associated with a significant improvement in ESS, in depression scale, and in four domains of HRQoL (energy/vitality, role emotional, role physical and social functioning). An improvement in three additional domains of HRQoL (general health, mental health and physical functioning) was observed in sleepy

TABLE 1 Outcomes of continuous positive airway pressure (CPAP) in non-sleepy and sleepy patients with obstructive sleep apnoea (OSA) after a mean \pm SD follow-up of $623 \pm 456$ days

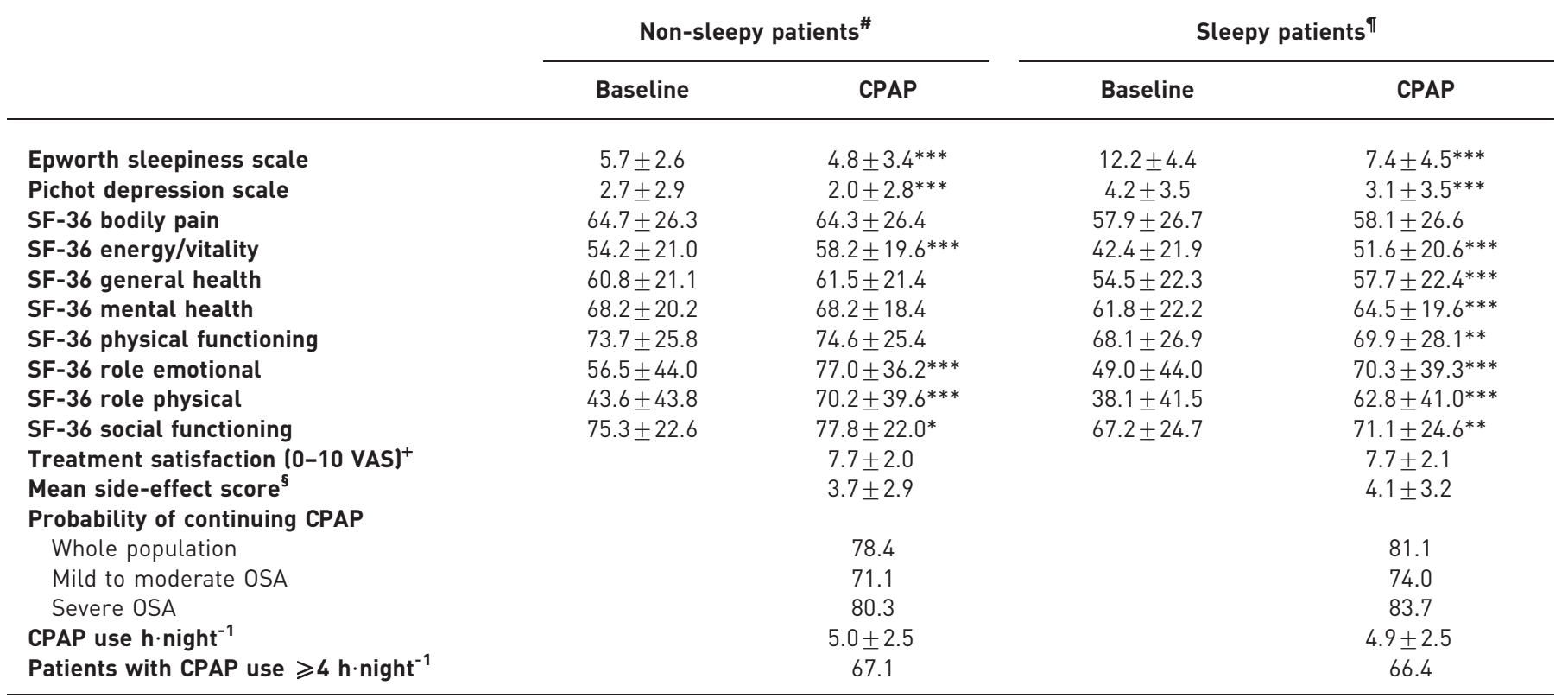

Data are presented as mean \pm SD or $\%{ }^{*}: \mathrm{n}=589 ;{ }^{\bullet}: \mathrm{n}=1799 ;{ }^{+}$: visual analog scale (VAS) from 0 (very dissatisfied) to 10 (very satisfied); ${ }^{\S}:$ patients were asked to give a score from 0-3 (0: absent, 1: mild, 2: moderate, 3: severe) concerning the six common side-effects of CPAP (congested, drippy or irritated nose, skin lesions, irritated eyes and dry mouth), a mean side-effect score from 0 to 18 was then calculated. *: $p<0.05$ versus baseline; ${ }^{* *}: \mathrm{p}<0.01$ versus baseline; ${ }^{* * *}: \mathrm{p}<0.001$ versus baseline. 
patients. Treatment satisfaction and mean CPAP side-effects score were similar in non-sleepy and sleepy patients. During follow-up, $466(19.5 \%)$ patients discontinued CPAP after a mean duration of $319 \pm 294$ days due to insufficient adherence (mean CPAP use $2.8 \pm 2.2 \mathrm{~h} \cdot$ night $^{-1}$ ). On Kaplan-Meier analysis, only mild and non-significant differences were observed between non-sleepy and sleepy patients regarding the probability of continuing CPAP over time in the whole population, in patients with mild-tomoderate OSA (AHI $<30, n=603$ ) and in patients with severe OSA (AHI $\geqslant 30, n=1785$ ). The average daily CPAP use recorded at each follow-up visit and the percentage of patients with mean daily CPAP use $\geqslant 4 \mathrm{~h}$ were also similar in non-sleepy and sleepy patients (table 1). On multivariable logistic regression analysis, including age, sex, BMI, AHI, depression, OSA symptoms, SES, CPAP mode and use of humidification, only AHI and the use of humidification were independently associated with CPAP dropout in non-sleepy patients. Adjusted odds ratios (95\% confidence intervals) for CPAP dropout were 1.82 (1.13-2.92) for AHI $<30$ versus $\mathrm{AHI} \geqslant 30$ and 1.72 (1.29-2.31) for no humidification versus humidification.

In line with previous data [4], the present study demonstrates that EDS is more strongly associated with depression than with OSA severity. These results suggest that patients with clinical suspicion of OSA and EDS should be also assessed for depression. Sleepy patients also had a higher BMI and were more likely to be diabetic, but the independent association between EDS and metabolic disorders was not confirmed by the multivariable analysis. Education level may influence self-reported symptoms awareness and perception [5]. Although the direction of the relationship and the underlying mechanisms were not established in the present study, our findings suggest that a lower level of education is independently associated with a lower complaint of EDS in OSA.

It is often postulated that non-sleepy OSA patients are not good candidates for CPAP and will probably present poor adherence. However, previous studies evaluating the influence of EDS prior to treatment on CPAP adherence have reported conflicting results $[1,2]$. The lack of interaction between OSA severity and sleepiness with regard to related cardiovascular morbidity suggests treating OSA patients with and without perceived EDS [6]. The vast majority of OSA patients without complaint of EDS were also found to exhibit attention defects that may potentially expose them to specific risks of accident during driving [7]. In line with recent findings from randomised controlled trials evaluating the impact of CPAP on cardiovascular outcomes in minimally symptomatic patients $[8,9]$, we demonstrate that long-term CPAP therapy is highly feasible and associated with an improvement in depression scores and HRQoL, in patients with an apparent paucity of symptoms at diagnosis. We also found that AHI is the only independent predictor of CPAP adherence prior to treatment in non-sleepy OSA patients. Altogether, our findings and those of recent randomised controlled trials [7,8] indicate that the distinction between "OSA" and "OSA syndrome (OSAS)" may not be clinically relevant, since almost 460 patients that would not qualify for the definition of syndrome complied with CPAP and perceived treatment benefit. Our findings also suggest that the use of humidification may contribute to improving CPAP adherence.

One potential limitation of the present study is its observational design. However, rigorous observational cohort studies can complement randomised controlled trial data with information on long term treatment efficacy and adherence in patients encountered in day-to-day clinical practice [10]. The present study can be assumed to describe a "typical" pattern of OSA patients, as this multisite study included a large sample of patients with a wide range of disease severity. However, our study was carried out only in patients referred with clinical suspicion of OSA and some patients with AHI $<30$ were not prescribed CPAP according to the French guidelines. CPAP adherence and self-assessed benefit should, therefore, not be extrapolated to all subjects with undiagnosed OSA.

Despite these potential limitations, the present study demonstrates that CPAP therapy is highly acceptable and associated with self-reported benefits in non-sleepy OSA patients referred for sleep study. Our findings do not support the use of EDS measured by ESS and/or subjective assessment as a selection criterion for CPAP therapy in OSA patients.

@ERSpublications

CPAP therapy is highly acceptable and associated with self-reported benefits in non-sleepy OSA patients http://ow.ly/my739

Frédéric Gagnadoux ${ }^{1,2,3}$, Marc Le Vaillant ${ }^{4}$, Audrey Paris ${ }^{5}$, Thierry Pigeanne ${ }^{6}$, Sylvaine Chollet $^{7}$, Philippe Masson ${ }^{8}$, Acya Bizieux-Thaminy ${ }^{9}$, Marie-Pierre Humeau ${ }^{10}$, Nicole Meslier ${ }^{1,2,3}$ and The IRSR sleep cohort group

${ }^{1}$ Lunam Université, Angers, ${ }^{2}$ Université d'Angers, CHU Angers, Département de Pneumologie, Angers, ${ }^{3}$ Inserm U1063, Angers, ${ }^{4}$ CERMES, CNRS UMR8211 - INSERM U988 - EHESS, Villejuif, ${ }^{5}$ Centre Hospitalier, Service de Pneumologie, Le Mans, ${ }^{6}$ Pôle santé des Olonnes, Unité de Pneumologie, Olonne sur Mer, ${ }^{7}$ Institut du Thorax, Pneumologie, Hôpital Laennec, Nantes, ${ }^{8}$ Centre Hospitalier, Service de Pneumologie, Cholet, ${ }^{9}$ Centre Hospitalier, Service de Pneumologie, La Roche sur Yon, and ${ }^{10}$ Nouvelles Cliniques Nantaises, Pneumologie, Nantes, France. 
Correspondence: Frédéric Gagnadoux, Département de Pneumologie, CHU, 4 rue Larrey, 49033 Angers Cedex 9, France. E-mail: frgagnadoux@chu-angers.fr

Received: Feb 262013 | Accepted: Mar 302013

Conflict of interest: Disclosures can be found alongside the online version of this article at www.ersjournals.com

Acknowledgements: The IRSR sleep cohort group members: F. Gagnadoux, N. Meslier, C. Person (Centre Hospitalier Universitaire, Angers); F. Goupil, O. Molinier (Centre Hospitalier, Le Mans); A. Bizieux-Thaminy, P. Breton, K. Berkani (Centre Hospitalier, La Roche sur Yon); T. Pigeanne (Pôle santé des Olonnes, Olonne sur Mer); S. Chollet, S. Jaffre, F. Corne, M. Boeffard, B. Nogues (Centre Hospitalier Universitaire, Nantes); M.P. Humeau (Nouvelles Cliniques Nantaises); C. Kierzkowski (M Normand de la Tranchade); J.L. Racineux, C. Gosselin (ALTADIR); M. Le Vaillant, N. Pelletier-Fleury (CERMES, CNRS UMR8211 - Inserm U988 - EHESS, Site CNRS).

\section{References}

McArdle N, Devereux G, Heidarnejad H, et al. Long-term use of CPAP therapy for sleep apnea/hypopnea syndrome. Am J Respir Crit Care Med 1999; 159: 1108-1114.

2 Gagnadoux F, Le Vaillant M, Goupil F, et al. Influence of marital status and employment status on long-term adherence with continuous positive airway pressure in sleep apnea patients. PLoS One 2011; 6: e22503.

3 Trzepizur W, Le Vaillant M, Meslier N, et al. Independent association between nocturnal intermittent hypoxemia and metabolic dyslipidemia. Chest 2013; 143: 1584-1589.

4 Bixler EO, Vgontzas AN, Lin HM, et al. Excessive daytime sleepiness in a general population sample: the role of sleep apnea, age, obesity, diabetes, and depression. J Clin Endocrinol Metab 2005; 90: 4510-4515.

5 McKee MM, Winters PC, Fiscella K. Low education as a risk factor for undiagnosed angina. J Am Board Fam Med, 25: 416-421.

6 Young T, Finn L, Peppard PE, et al. Sleep disordered breathing and mortality: eighteen-year follow-up of the Wisconsin sleep cohort. Sleep 2008; 31: 1071-1078.

7 Mazza S, Pépin JL, Naëgelé B, et al. Most obstructive sleep apnoea patients exhibit vigilance and attention deficits on an extended battery of tests. Eur Respir J 2005; 25: 75-80.

8 Barbé F, Durán-Cantolla J, Sánchez-de-la-Torre M, et al. Effect of continuous positive airway pressure on the incidence of hypertension and cardiovascular events in nonsleepy patients with obstructive sleep apnea: a randomized controlled trial. JAMA 2012; 307: 2161-2168.

9 Craig SE, Kohler M, Nicoll D, et al. Continuous positive airway pressure improves sleepiness but not calculated vascular risk in patients with minimally symptomatic obstructive sleep apnoea: the MOSAIC randomised controlled trial. Thorax 2012; 67: 1090-1096.

10 Silverman SL. From randomized controlled trials to observational studies. Am J Med 2009; 122: 114-120.

\section{Severe acute respiratory distress syndrome due to ipilimumab}

To the Editor:

Ipilimumab is a T-cell-potentiating human monoclonal antibody against cytotoxic T-lymphocyteassociated antigen (CTLA)-4, which promotes antitumour immunity by unrestrained T-cell activation [1]. It is the first agent shown to improve survival in patients with metastatic melanoma [2]. However, various ipilimumab-associated immune-related adverse events including enterocolitis, dermatitis, hepatitis, hypophysitis, meningo-radiculo-neuritis, and renal failure have been reported [3]. The few reported cases of pulmonary side-effects consist of ipilimumab-induced sarcoid-like granulomatosis [4-7]. To our knowledge, this is the first report of an ipilimumab-induced lung injury presenting as severe acute respiratory distress syndrome (ARDS).

A 64-year-old former smoker with metastatic melanoma (stage IIIC) was referred with hypoxaemic respiratory failure and oedema of both feet and hands, following administration of the second dose of ipilimumab (246 mg) 4 days earlier. After the first dose $(237 \mathrm{mg})$, given 6 weeks earlier in combination with radiotherapy for inguinal lymph node metastases and topical immunotherapy with diphenylcyclopropenone $0.1 \%$ for cutaneous metastases, the patient presented with generalised exanthema in the absence of respiratory symptoms, which was responsive to oral and topical steroids. His past medical history was uneventful for pulmonary diseases, and he took no drugs other than ipilimumab and prednisone $\left(30 \mathrm{mg} \cdot \mathrm{day}^{-1}\right)$ for exanthema. He was tachycardic $\left(120 \mathrm{beats} \cdot \mathrm{min}^{-1}\right)$ and normotensive. His respiratory rate was 34 per min and a peripheral cyanosis was present. The jugular veins were not distended, but marked oedema of both the feet and hands were present. Cardiac auscultation was unrevealing, but bilateral crackles 